\title{
THE IMPROVEMENT OF PRODUCTION USING ELEMENTS OF THE TOYOTA'S HOUSE ROOF - CASE STUDY
}

doi: $10.2478 /$ czoto-2020-0041

Date of submission of the article to the Editor: $15 / 11 / 2019$

Date of acceptance of the article by the Editor: 15/12/2019

Krzysztof Mielczarek ${ }^{1}$ - orcid id: 0000-0003-3701-0192

${ }^{1}$ Czestochowa University of Technology, Poland

\begin{abstract}
The article presents a case study of the practical use of BOST surveys to identify the most important factors in chosen enterprise. This article presents an analysis of the answers given to the question contained in the BOST questionnaire, referring to the roof of Toyota's house. Toyota's management principles by Jeffrey Liker were pointed out, which refers to elements of Toyota's house roof. Results of the survey were presented and the proposal to improving the processes in the enterprise. Research results were subjected to statistical analysis with a use of basic statistical parameters. It was made a correlation analysis connected with elements of the Toyota's house roof. Evaluation structure was presented in a graphic form. Based on the survey results of carried out on the population of production workers, a series of importance areas for improvement was formulated. The aim of the analysis is to present which factors are the most important by building the significance sequences of obtained results.The results obtained for the type of small and medium-sized enterprises overlap with the results of tests verified in other enterprises.
\end{abstract}

Keywords: BOST method, Toyota's house roof, importance hierarchy, statistical analysis, improvement

\section{INTRODUCTION - METHODOLOGY OF RESEARCH AND PRESENTATION OF RESULTS}

The object of the survey is an enterprise which manufactures different type of groceries such as juices and drinks - production of nectars, juices and drinks about and large number of different tastes, food concentrates - mainly a production of concentrate and the apple aroma, concentrate of the black currant and wide assortment of canned vegetables for the export and local market. The enterprise owns a wellequipped machine park, which enabled to create many products ordered by customers.

Survey and research method determined as BOST (the name of the is an acronym created from the first two letters of the name and surname of his creator i.e. Stanislaw Borkowski, professor of technical and economic sciences and the acronym is legally protected) was formed as a result of author's fascination in Toyota Motor Company (Borkowski, 2016). The BOST method defines relations between material resources 
and human resources and be-tween human resources and human resources and is named Toyotarity (Knop, 2015). The BOST method describes Toyota's management principles with its characteristic factors. The set of factors is called an area. Each of these principles described with an appropriate set of factors. Apart from Toyota's principles described with applied factors and respondents' features the set of research problems also contains elements of the roof of Toyota's house, which describe the mission of enterprises (Amasaka, 2012, Kehr and Proctor, 2017). Toyota's management principles are divided into sections that contains a set of factors describing principles: $1 ; 2 ; 3 ; 4 ; 6 ; 7 ; 14$ and elements of the roof of Toyota's house (Gao and Low, 2015). The presented questionnaire has a ranking scale (Liker and Franz, 2011; Liker and Hoseus, 2008). Respondents may assess the significance of a given factor by placing one of the numbers within the range of scale in an appropriate box. A preliminary condition for classification of the companies to BOST study was confirmed information about implementation of Toyota management principles in the workstations in analyzed company. In selected enterprise the population of respondents was chosen, which consisted from production workers of the examined enterprise, having a contact with manufacturing process in the workplace.

The BOST questionnaire form was filled out, by 30 respondents i.e. nearly half of production workers. Such a large research group of directly production workers will allow to precise identification the most important factors in the surveyed enterprise. This article presents an analysis of the answers given to the question contained in the BOST questionnaire, referring to the roof of Toyota's house. In the purpose to form an opinion it is essential to know the opinion of workers from different ranks in enterprise. It lets on better look on the enterprise by eyes of workers. BOST is survey where the questions are so well-matched as to judge enterprise and its immaterial stores are possible (Mielczarek, 2015). This article presents an analysis of the answers given to the question contained in the BOST questionnaire, referring to the roof of Toyota's house (Borkowski et al., 2014; Selejdak, 2015). Employees have answered the following question: "Which factor is the most important in your enterprise?" Fill in the blanks with $1 ; 2 ; 3 ; 4 ; 5$ (5 the most important factor):

\begin{tabular}{|c|l|l}
\hline JA & & $\begin{array}{l}\text { Quality } \\
\text { Cost }\end{array}$ \\
\cline { 1 - 2 } KO & & $\begin{array}{l}\text { Execution time } \\
\text { Work safety }\end{array}$ \\
\cline { 1 - 1 } BP & & Attitude of the crew \\
\cline { 1 - 2 } MZ & &
\end{tabular}

Table 1 present a percentage list of significance rates of Toyota's house roof area factors in the enterprise producing different type of drinks and juices.

Table 1

Elements of the Toyota's house roof. Percentage list of factors' significance rates

\begin{tabular}{|c|c|c|c|c|c|}
\hline \multirow{2}{*}{ Evaluation } & \multicolumn{5}{|c|}{ Indicating the factors } \\
\cline { 2 - 6 } & JA & KO & CR & BP & MZ \\
\hline $\mathbf{1}$ & 0 & 10.0 & 13.3 & 20.0 & 56.6 \\
\hline $\mathbf{2}$ & 3.3 & 63.3 & 13.3 & 13.3 & 6.7 \\
\hline $\mathbf{3}$ & 6.7 & 6.7 & 40.0 & 16.7 & 30.0 \\
\hline $\mathbf{4}$ & 26.7 & 3.3 & 33.3 & 30.0 & 6.7 \\
\hline $\mathbf{5}$ & 63.3 & 16.7 & 0 & 20.0 & 0 \\
\hline
\end{tabular}

Source: own study 
Data presented in the above-mentioned table prove that employees of the examined enterprise consider quality (JA) as the most important element of the company. The results contained in Table 1 were graphically presented with the use of radar charts.

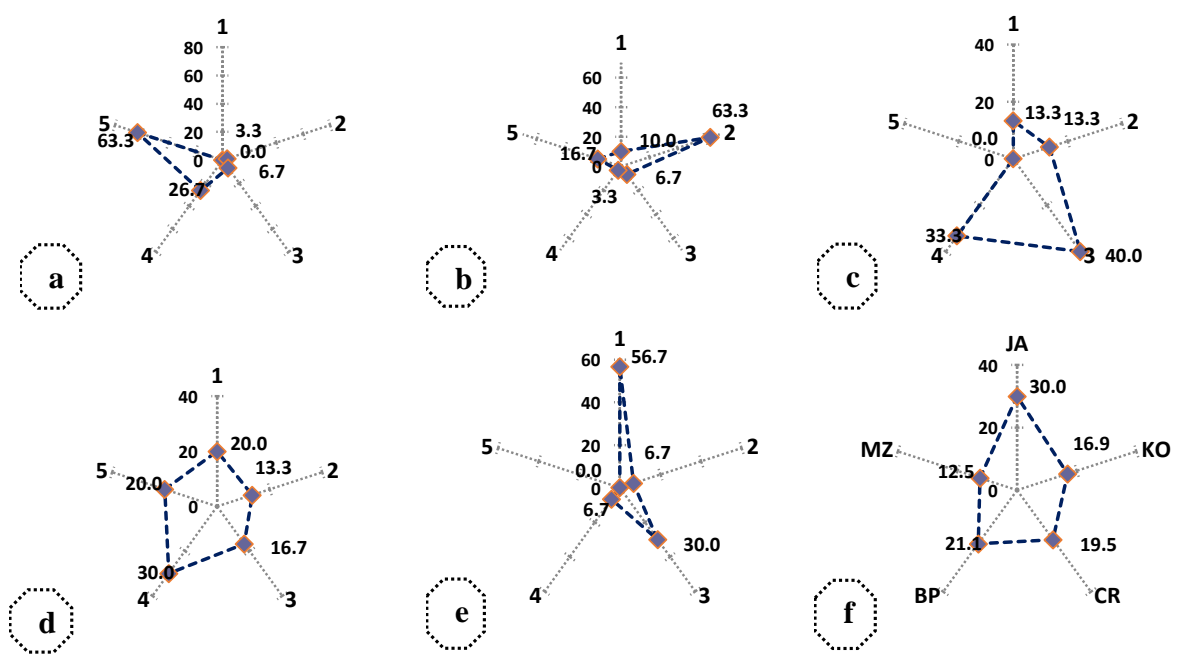

Fig. 1. Radar charts - percentage structure for ratings: a) JA, b) KO, c) CR, d) BP,

e) $M Z, f)$ average

Radar charts show the structure of average rates for factors describing the elements of the Toyota's house roof. Based on the prepared structure of ratings for separate fac-tors it was found that quality (JA) was the most important factor for $63.3 \%$ of respondents. They have awarded that factor the highest rate " 5 ". In the case of the next factor, cost (KO) the answers were different, because $16.7 \%$ have granted this factor the highest rate " 5 " while for other respondents $(63.3 \%)$ was less important and they have granted it rate "2". Execution time (CR) has been assessed by respondents as a not significant factor. The next factor was work safety (BP). 20\% of respondents gives this factor rate " 5 ", for $20 \%$ of them this factor has been far less significant because it has been assessed on the level "1". Attitude of the crew (MZ) has been assessed as follows: none of the respondents has granted it rate " 5 ", only $6.7 \%$ have given rate " 4 " to this factor, whereas for $56.6 \%$ of respondents this factor has not been significant at all. Numerical distribution of individual evaluations is presented in Fig. 2.

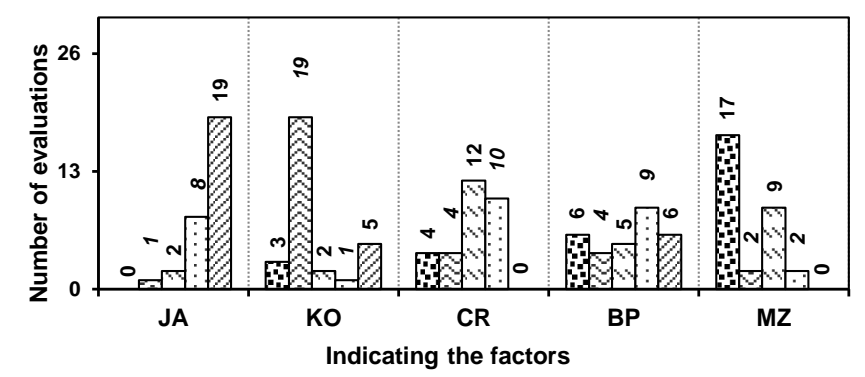

Fig. 2. Roof of the Toyota's house. Numerical distribution of individual evaluations for factors

\section{CORRELATION ANALYSIS OF THE INFLUENCE OF RESPONDENTS' CHAR- ACTERISTIC FEATURES ON THE SIGNIFICANCE RATES OF ENTERPRISE MIS- SION FACTORS}

Respondents differ in terms of their personal features: gender, education, age, work experience, mobility, mode of employment. To determine the influence of these fea- 
tures, correlation coefficients were calculated and presented in the form of graphs in Fig. 3. Each of these Figures also contains three characteristic lines on each side of the 0 axis. It should be noted that their distance from the 0 axis is the same in all the Figures, because they represent the value of a critical coefficient for various levels $\alpha$ $(0.05 ; 0.10 ; 0.2)$. In the analyzed enterprise the influence of respondents' characteristic features on the significance of factors of enterprises' mission was defined with use of the correlation analysis (Knop, 2018).
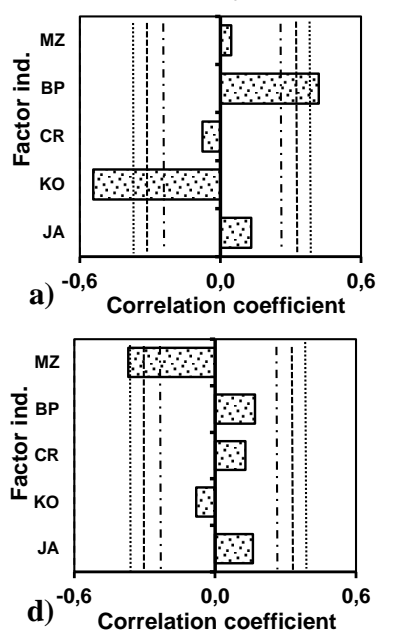
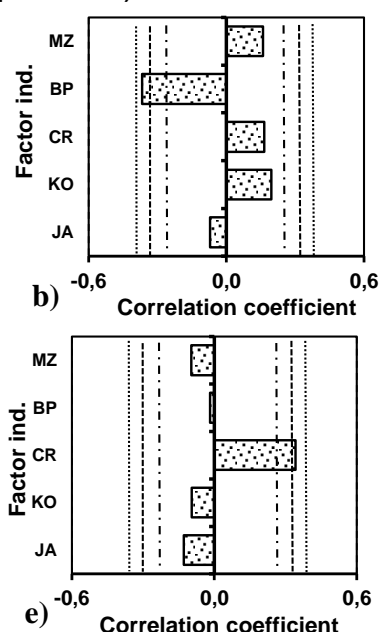
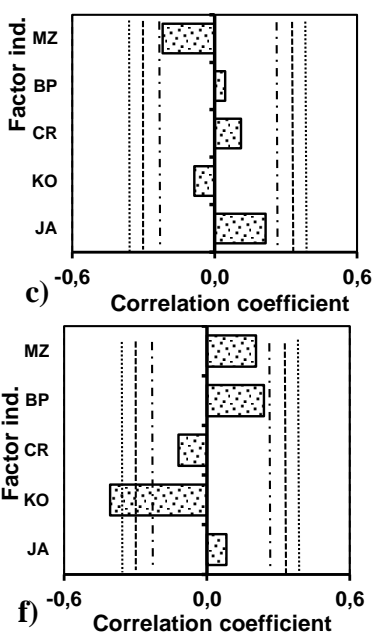

Fig. 3. Elements of Toyota's house roof. Correlation graphs for factors of Toyota's house roof area according to the characteristics of respondents: a) gender, b) education, c) age, d) work experience, e) mobility, f) mode of employment. $\alpha=0.2$ (internal lines), $\alpha=0.1$ (middle line), $\alpha$ $=0.05$ (external lines)

The analysis allows for drawing the following conclusions: it was found that not each feature of respondent had influence on the level of rates. It is possible to state that the gender is feature of respondents which is entering correlations with two factors. Examining the relation be-tween education of respondents we can notice that in this case appeared negative correlation for work safety (BP). Age have no significant influence on the importance of any factor of the analyzed area. Correlation between work experience and importance of attitude of the crew (MZ) is significant for all $\alpha$ level. In the case of the relation between the mobility and factors it was noticed that the mobility correlating (positively) with only one factor execution time (CR). Mode of employment has influence on the assessment of cost (KO).

\section{CORRELATION STATISTICAL ANALYSIS OF THE RESULTS FROM THE BOST QUESTIONNAIRE}

Making statistical analysis of studied area six statistical tools were used: arithmetic average, variance, standard deviation, the coefficient of variation, skewness and kurtosis (Fig. 4). The aim of application of this statistical tool is to show distribution of evaluation for individual factors.

The average level of the measurable feature was presented with the help of the average. Analyzing Fig. 4a concerning the result of average it was taken the conclusion that majority of respondents judged the response concerning quality (JA) on the level 4.50. The lowest average rate has been acquired by attitude of the crew (MZ), which has been assessed on the level 1.87 on aver-age. 
The next statistical tool is a variance (Knop and Mielczarek, 2018). From Fig. 4b results that the maximum value of variance amounting 2.07 concerning reply work safety (BP). Minimum value 0.60 received the answer quality (JA).

Standard deviation (Fig. 4c) is the biggest for the factor work safety (BP) - on the level 1.44 and the smallest for quality $(\mathrm{JA})-0.78$.

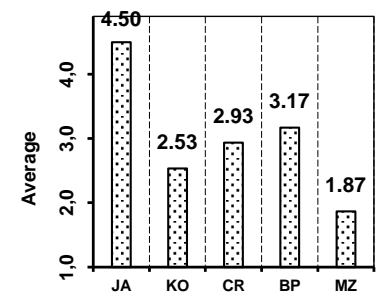

a) Indicating the factors

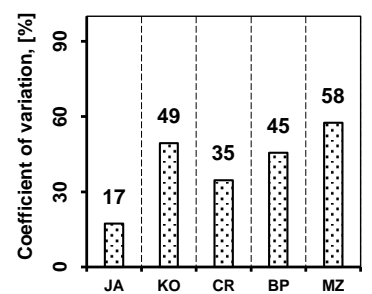

d)

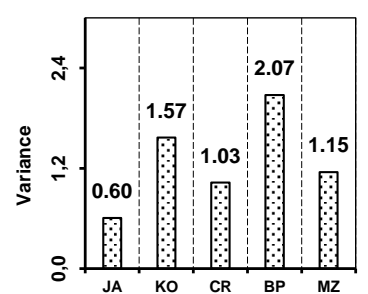

b) Indicating the factors

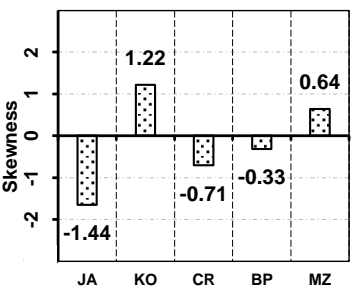

e)

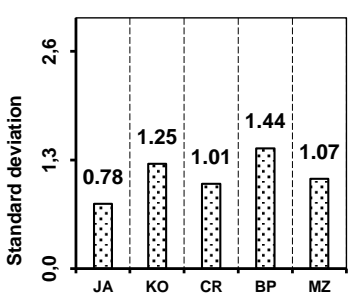

c) Indicating the factors

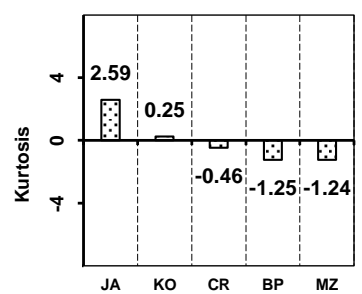

f)

Indicating the factors

Fig. 4. Comparison of: a) average values, b) standard deviation, c) variance, d) variation coefficient, e) skewness, f) kurtosis for E6 area factors

In order to assess factors with the use of variation coefficient it is necessary to prepare the following statement: $0-20 \%$ - weak variation of feature, $20-40 \%$ - moderate variation of feature, 40 - 60\% - strong variation of feature, $60 \%$ and more - very strong variation of feature (Borkowski and Ulewicz, 2009, Wheeler, 2000). Coefficients of variation presented at Fig. $4 \mathrm{~d}$ show, a weak variation for quality (JA), a strong variation of factors: cost $(\mathrm{KO})$, work safety $(\mathrm{BP})$ and attitude of the crew $(\mathrm{MZ})$. The measurement of skewness is (Fig. 4e) a classic co-efficient of asymmetry: (0.0 $0.4)$ - very weak distribution asymmetry, (0.4 - 0.8) - weak distribution asymmetry, (0.8 - 1.2) - moderate distribution asymmetry, (1.2 - 1.6) - strong distribution asymmetry, (more than 1.6 - very strong distribution asymmetry) (Fouad and Mukat-tash, 2010). The distribution of rates for quality (JA) indicate strong negative skewness and for factor cost (KO) indicate strong positive skewness. The distribution of rates for the rest of factors indicates weak skewness.

The last factor for analyzing is kurtosis (Fig. 4f). It determines the measure of distribution and concentrating the results in surroundings of the average (Ignaszak and Sika, 2012; Uçurum et al., 2016). For appropriate interpretation of results the following statement is necessary: We $<0$ - distribution is characterized by lower than standard peakedness, $\mathrm{We}=0$ distribution is characterized by standard peakedness, We $>0-$ distribution is characterized by peakedness higher than standard. For factors quality (JA) and cost (KO) the distribution of rates is characterized by higher than standard peakedness.

This statistical tool confirm that distribution of results is logical and can be helpful for evaluation actual state in enterprise. 


\section{Summary}

Surveyed studies have shown the effectiveness of the selection of specific factors in the purpose of determining a series of validity. The use of this knowledge will enable effective use of the company's resources in improving the indicated areas. As a result of the research work, it was found that the most important area requiring improvement is quality. It is an important element of research for small and medium enterprises. The results of research are consistent with the research carried out in other such enterprises.

By way of conclusion was presented importance series of factors for average evaluations (Fig. 5). Summing up, a range of important factors in examined enterprise is following:

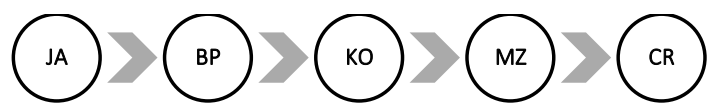

Fig. 5. The importance series of the factors for elements of Toyota's house roof

From analysis of the averages of important factors it results that quality (JA) is a key factor according to respondents (among examined factors it is on the second place in a range of important factors).

Data obtained from BOST analysis allowed to know the opinions of representative group of workers in the topic of functioning of the enterprise concerning the competent organization of a production process and its influence on the quality of produced goods. Functioning of the production system, although now is working perfectly, it is possible still to be improved. The BOST questionnaire survey allowed to obtain the information what factors are the most important for the enterprise workers in the production process.

How results from the BOST questionnaire survey, that factor quality (JA) often has been judged with the highest evaluation by respondents. The second position was taken by the factor safety in the workplace (BP). Studies showed that safety in a range of important factors of the production process took important place, which indicates its significance in the production process in company producing juices and drinks. In the researched company a quality of products, as well as safety in the workplace are important in production process and in the entire enterprise. Significance sequences of factors describing roof of the Toyota's house is an interesting element. They contain a logical order conditioned by organization of production in the company.

A production a reliable, functional and safe products is the key to success. Functioning of the production system, although now is working perfectly, it is possible still to be improved. The quality of offered products can be improved through applying tools of the quality management.

The practical use of BOST surveys gives the opportunity to benefit from the practical knowledge of employees at the company's production level. This may contribute to the identification of key areas for the functioning of the enterprise.

\section{REFERENCES}

Amasaka, K., 2012. Science TQM, New Quality Management Principle, The Quality Management Strategy of Toyota Introduction, Bentham Sc., U Arab Emirates. 
Borkowski, S., 2016. Scientific Potential of Toyotarity and BOST Method, Polish Quality Institute, Warsaw.

Borkowski, S., Jagusiak-Kocik, M., Ingaldi, M., 2014. Evaluation of the Manufacturing of Components for Combine Harvesters Using Bost Method, 23nd International Conference on Metallurgy and Materials METAL, Brno.

Fouad, R.H., Mukattash, A., 2010. Statistical Process Control Tools: A Practical guide for Jordanian Industrial Organizations, Jordan Journal of Mechanical and Industrial Engineering, 4, 6, 693-700.

Gao, S., Low, S.P., 2015. Toyota way style human resource management in large Chinese construction firms. A qualitative study, International Journal of Construction Management, 15(1), 17-32.

Ignaszak, Z., Sika, R., 2012. Specificity of SPC Procedures Application in Foundry in Aspect of Data Acquisition and Data Exploration, Archives of Foundry Engineering, 12(4,) 65-70, DOI: 10.2478/v10266-012-0108-8

Kehr, TW., Proctor, MD., 2017. People Pillars: Re-structuring the Toyota Production System (TPS) House Based on Inadequacies Revealed During the Automotive Recall Crisis. Quality And Reliability Engineering International, 33(4), 921-930, DOI: 10.1002/qre.2059

Knop, K., 2015. Statistical Analysis of Responses Concerning the Importance of Human and Production or Services Issues in Various Companies, Production Engineering Archives, 7/2(2015), 40-44, DOI: 10.30657/pea.2015.07.10

Knop, K., 2018. Statistical Control of the Production Process of Rolled Products, Production Engineering Archives, 20(2018), 26-31. DOI: 10.30657/pea.2018.20.06

Knop, K., Mielczarek, K., 2018. Assessment of Production Processes Functioning in the Case of Air Bag Production, MATEC Web Conf. 183, 04009, DOI: 10.1051/matecconf/201818304009

Liker, J.K., Franz, J., 2011. The Toyota Way to continuous improvement, McGraw Hill.

Liker, J.K., Hoseus, M., 2008. Toyota Culture: The Heart and Soul of the Toyota Way. McGraw-Hill, New York.

Mielczarek, K., 2015. Factors Describing the Concept of Plastics Company Development, Production Engineering Archives, 8/3(2015), 32-35., DOI: DOI: 10.30657/pea.2015.08.08

Selejdak, J., 2015. Use of the Toyota Management Principles for Evaluation of the Company's Mission, Production Engineering Archives, 1/1(2013), 13-15, DOI: 10.30657/pea.2013.01.04

Uçurum, M., Çolak, M., Çınar, M., Dışpınar, D., 2016. Implementation of Statistical Process Control (SPC) Techniques as Quality Control in Cast Iron Part Production, Journal of Engineering Precious Research and Application, 1(3), 14-24.

Wheeler, D., 2000. Understanding Variation: The Key to Managing Chaos, 2 Revised Edition, SPC Press. 